\title{
A quick proof of nonvanishing for asymptotic syzygies
}

\author{
Lawrence Ein, Daniel Erman and Robert Lazarsfeld
}

\begin{abstract}
We give a quick new approach to proving the main cases of the nonvanishing theorems of the first and third authors concerning the asymptotic behavior of the syzygies of a projective variety as the positivity of the embedding line bundle grows. In particular, we recover and extend the statements for Veronese syzygies, and we establish an effective result for projectively Cohen-Macaulay varieties.
\end{abstract}

\section{Introduction}

The purpose of this note is to give a very quick new approach to the main cases of the nonvanishing theorems of [EL12] concerning the asymptotic behavior of the syzygies of a projective variety as the positivity of the embedding line bundle grows. In particular, we present a surprisingly elementary and concrete approach to the asymptotic nonvanishing of Veronese syzygies, and we obtain effective statements for arithmetically Cohen-Macaulay varieties.

Let $X$ be an irreducible projective variety of dimension $n$ over an algebraically closed field $\mathbf{k}$, and let $L$ be a very ample divisor on $X$, defining an embedding

$$
X \subseteq \mathbf{P} H^{0}(L)=\mathbf{P}^{r} .
$$

Write $S=\operatorname{Sym}\left(H^{0}(L)\right)$ for the homogeneous coordinate ring of $\mathbf{P}^{r}$, and for a fixed divisor $B$ on $X$ consider the $S$-module

$$
M=M(B ; L)=\bigoplus_{m} H^{0}(B+m L)
$$

We are interested in the minimal graded free resolution $E_{\bullet}=E_{\bullet}(B ; L)$ of $M$ over $S$ :

$$
0 \longrightarrow E_{r} \longrightarrow \ldots \longrightarrow E_{1} \longrightarrow E_{0} \longrightarrow M \longrightarrow 0
$$

with $E_{p}=\oplus S\left(-a_{p, j}\right)$. Denote by

$$
K_{p, q}(B ; L)=K_{p, q}(X, B ; L)
$$

the finite-dimensional vector space of degree $p+q$ minimal generators of the $p$ th module of syzygies of $M$, so that

$$
E_{p}(B ; L)=\bigoplus_{q} K_{p, q}(B ; L) \otimes_{k} S(-p-q)
$$

Received 19 January 2015, accepted in final form 4 September 2015.

2010 Mathematics Subject Classification 14J99, 13 D02.

Keywords: syzygies, free resolutions, very positive embeddings.

This journal is (C) Foundation Compositio Mathematica 2016. This article is distributed with Open Access under the terms of the Creative Commons Attribution Non-Commercial License, which permits non-commercial reuse, distribution, and reproduction in any medium, provided that the original work is properly cited. For commercial re-use, please contact the Foundation Compositio Mathematica.

Research of the first author partially supported by NSF grant DMS-1001336. Research of the second author partially supported by NSF grant DMS-1302057. Research of the third author partially supported by NSF grant DMS-1439285. 


\section{Ein, D. ERman and R. LAzArsfeld}

(When $B=\mathcal{O}_{X}$, we write simply $K_{p, q}(X ; L)$ or $K_{p, q}(L)$ if no confusion seems likely.) It is elementary that if $L$ is very positive compared to $B$, then nonzero syzygies can only appear in weights $0 \leqslant q \leqslant n+1$, and it turns out that the extremal cases $q=0$ and $q=n+1$ are easy to control. So the first interesting question is to fix $B$ and $1 \leqslant q \leqslant n$, and to ask which groups $K_{p, q}(B ; L)$ are nonvanishing when $L$ becomes very positive. The main result of [EL12] asserts in effect that - contrary to what one might have expected by extrapolating from the case of curves - these groups are eventually nonzero for almost all values of $p \in[1, r]$.

Perhaps the most natural instance of these matters occurs when $X=\mathbf{P}^{n}, B=\mathcal{O}_{\mathbf{P}^{n}}(b)$, and $L=L_{d}=\mathcal{O}_{\mathbf{P}^{n}}(d)$, so that one is looking at the syzygies of Veronese varieties. It was established in [EL12] that if one fixes $q \in[1, n]$ and $b \geqslant 0$, then for $d \gg 0$ one has

$$
K_{p, q}\left(\mathbf{P}^{n}, B ; L_{d}\right) \neq 0
$$

for every value of $p$ satisfying

$$
\left(\begin{array}{c}
d+q \\
q
\end{array}\right)-\left(\begin{array}{c}
d-b-1 \\
q
\end{array}\right)-q \leqslant p \leqslant\left(\begin{array}{c}
d+n \\
n
\end{array}\right)-\left(\begin{array}{c}
d+n-q \\
n-q
\end{array}\right)+\left(\begin{array}{c}
n+b \\
n-q
\end{array}\right)-q-1 .
$$

For example, when $n=2$ and $b=0$, this asserts that

$$
K_{p, 2}\left(\mathbf{P}^{2} ; \mathcal{O}_{\mathbf{P}^{2}}(d)\right) \neq 0 \quad \text { for } 3 d-2 \leqslant p \leqslant\left(\begin{array}{c}
d+2 \\
2
\end{array}\right)-3,
$$

which was the main result of the interesting paper [OP01] of Ottaviani and Paoletti. The proof in [EL12] of the Veronese nonvanishing theorem involved a rather elaborate induction on $n$ to show that certain well-chosen secant planes to the Veronese variety force the presence of nonzero syzygies. For $b=0$ the same statement was obtained independently in characteristic zero by Weyman, who identified certain representations of SL $(n+1)$ that appear nontrivially in the $K_{p, q}$. Some other work concerning Veronese syzygies appears in [Rub04, BCR11b, BCR11a, EGHP05], and a simplicial analogue of the results of [EL12] is given in [CJKW14].

The goal of the present paper is to present a much simpler and more elementary approach to the nonvanishing of Veronese syzygies, and to use this method to establish effective statements for arithmetically Cohen-Macaulay varieties. The idea is that one can reduce the question to elementary computations with monomials by working modulo a suitable regular sequence. In order to explain how this goes, consider the problem of proving the first case of the OttavianiPaoletti statement (1.2), namely that if $d \geqslant 3$, then

$$
K_{3 d-2,2}\left(\mathbf{P}^{2} ; \mathcal{O}_{\mathbf{P}^{2}}(d)\right) \neq 0 .
$$

Writing $S_{k}$ for the degree $k$ piece of the polynomial $\operatorname{ring} S=\mathbf{k}[x, y, z]$, it is well known that the group in question can be computed as the cohomology at the middle term of the Koszul-type complex

$$
\ldots \longrightarrow \Lambda^{3 d-1} S_{d} \otimes S_{d} \longrightarrow \Lambda^{3 d-2} S_{d} \otimes S_{2 d} \longrightarrow \Lambda^{3 d-3} S_{d} \otimes S_{3 d} \longrightarrow \ldots
$$

The most naive approach to (1.3) would be to write down explicitly a cocycle representing a nonzero element in $K_{3 d-2,2}$, but we do not know how to do this. ${ }^{1}$ On the other hand, consider the ring

$$
\bar{S}=S /\left(x^{d}, y^{d}, z^{d}\right)
$$

As $x^{d}, y^{d}, z^{d}$ form a regular sequence in $S$, the dimensions of the Koszul cohomology groups of $\bar{S}$ are the same as those of $S$, and hence the question is equivalent to proving the nonvanishing of

${ }^{1}$ The argument in [OP01] proceeds by using duality to reformulate the question as the nonvanishing of a $K_{p^{\prime}, 0}$, where one can exhibit directly the required class. 


\section{A QUICK PROOF OF NONVANISHING}

the cohomology of

$$
\cdots \longrightarrow \Lambda^{3 d-1} \bar{S}_{d} \otimes \bar{S}_{d} \longrightarrow \Lambda^{3 d-2} \bar{S}_{d} \otimes \bar{S}_{2 d} \longrightarrow \Lambda^{3 d-3} \bar{S}_{d} \otimes \bar{S}_{3 d} \longrightarrow \cdots
$$

Now view $\bar{S}$ as the ring spanned by monomials in which all variables have exponent at most $d-1$, with multiplication governed by the vanishing of the $d$ th powers of each variable. The plentiful presence of zero-divisors in $\bar{S}$ means that one can write down by hand many monomial Koszul cycles: for instance if $m_{1}, \ldots, m_{3 d-2}$ are monomials of degree $d$, each divisible by $x$ or $y$, then

$$
c=m_{1} \wedge \cdots \wedge m_{3 d-2} \otimes x^{d-1} y^{d-1} z^{2}
$$

gives a cycle for the complex (1.4). Note next that $x^{d-1} y^{d-1} z^{2}$ has exactly $3 d-2$ monomial divisors of degree $d$ with exponents at most $d-1$, namely

$$
\begin{gathered}
x^{d-1} y, x^{d-2} y^{2}, \ldots, x^{2} y^{d-2}, x y^{d-1}, \\
x^{d-1} z, x^{d-2} y z, \ldots, x y^{d-2} z, y^{d-1} z, \\
x^{d-2} z^{2}, x^{d-3} y z^{2}, \ldots, x y^{d-3} z^{2}, y^{d-2} z^{2} .
\end{gathered}
$$

Taking these as the $m_{i}$, we claim that the resulting cycle $c$ represents a nonzero Koszul cohomology class. In fact, suppose that $c$ appears even as a term in the Koszul boundary of an element

$$
e=n_{0} \wedge n_{1} \wedge \cdots \wedge n_{3 d-2} \otimes g,
$$

where the $n_{i}$ and $g$ are monomials of degree $d$. After re-indexing and introducing a sign we can suppose

$$
c=n_{1} \wedge \cdots \wedge n_{3 d-2} \otimes n_{0} g .
$$

Then the $\left\{n_{j}\right\}$ with $j \geqslant 1$ must be a re-ordering of the monomials $\left\{m_{i}\right\}$ dividing $x^{d-1} y^{d-1} z^{2}$. On the other hand $n_{0} g=x^{d-1} y^{d-1} z^{2}$, so $n_{0}$ is also such a divisor. Therefore $n_{0}$ coincides with one of $n_{1}, \ldots, n_{3 d-2}$, and hence $e=0$, giving a contradiction.

We show that this sort of argument gives the nonvanishing of Veronese syzygies appearing in equation (1.1), as well as a few further cases that were conjectured in [EL12]. Moreover, we obtain a new statement that subsumes the previous statement and includes all values of $b, q$, and $d$ (Theorem 2.1). More interestingly, whereas the results of [EL12] for varieties other than $\mathbf{P}^{n}$ were ineffective, we are able here to give effective statements for a large class of general varieties.

Specifically, consider an arithmetically Cohen-Macaulay variety $X \subseteq \mathbf{P}^{m}$ of dimension $n$, and for $d>0, b \geqslant 0$ write

$$
L_{d}=\mathcal{O}_{X}(d), \quad B=\mathcal{O}_{X}(b) .
$$

Put $c(X)=\min \left\{k \mid H^{n}\left(X, \mathcal{O}_{X}(k-n)\right)=0\right\}$, the Castelnuovo-Mumford regularity of $\mathcal{O}_{X}$, and write

$$
r_{d}=\operatorname{dim} H^{0}\left(X, \mathcal{O}_{X}(d)\right), \quad r_{d}^{\prime}=r_{d}-(\operatorname{deg} X)(n+1) .
$$

We prove the following result.

Theorem. Let $q \in[1, n-1]$, and fix $d \geqslant b+q+c(X)+1$. Then

$$
K_{p, q}\left(X, B ; L_{d}\right) \neq 0
$$

for every value of $p$ satisfying

$$
\operatorname{deg}(X)(q+b+1)\left(\begin{array}{c}
d+q-1 \\
q-1
\end{array}\right) \leqslant p \leqslant r_{d}^{\prime}-\operatorname{deg}(X)(d-q-b)\left(\begin{array}{c}
d+n-q-1 \\
n-q-1
\end{array}\right) .
$$




\section{Ein, D. ERman And R. LAZARSFeld}

Analogous statements hold, with slightly different numbers, when $q=0$ and $q=n$; see Theorem 3.1 below. We note that Zhou [Zho14] has given effective results for adjoint-type (and in particular, for very positive) line bundles $B$ on an arbitrary smooth complex projective variety. It would be interesting to know whether one could recover his statement by the present techniques; see Remark 3.7.

\section{Nonvanishing results for $\mathrm{P}^{n}$}

This section is devoted to the nonvanishing results for Veronese syzygies.

Let $\mathbf{k}$ be any field, and consider the polynomial $\operatorname{ring} S=\mathbf{k}\left[x_{0}, \ldots, x_{n}\right]$. Given $d \geqslant 1$ we denote by $S^{(d)} \subseteq S$ the Veronese subring

$$
S^{(d)}=\bigoplus_{j \in \mathbf{Z}} S_{j d} \subseteq S
$$

of $S$. For an $S$-module $M$, we write $M(b)^{(d)}$ for the $S^{(d)}$-module $\bigoplus_{j \in \mathbf{Z}} M_{b+j d}$. Note that $M(b)^{(d)}$ is also naturally a $\operatorname{Sym}\left(S_{d}\right)$-module. We denote by

$$
K_{p, q}(n, b ; d)=K_{p, q}^{\operatorname{Sym}\left(S_{d}\right)}\left(S(b)^{(d)}\right)
$$

the Koszul cohomology group of $S(b)^{(d)}$, where $S(b)^{(d)}$ is considered as a $\operatorname{Sym}\left(S_{d}\right)$-module. Thus $K_{p, q}(n, b ; d)$ is the cohomology of the Koszul-type complex

$$
\ldots \longrightarrow \Lambda^{p+1} S_{d} \otimes S_{(q-1) d+b} \longrightarrow \Lambda^{p} S_{d} \otimes S_{q d+b} \longrightarrow \Lambda^{p-1} S_{d} \otimes S_{(q+1) d+b} \longrightarrow \ldots
$$

and

$$
K_{p, q}(n, b ; d)=K_{p, q}\left(\mathbf{P}^{n}, \mathcal{O}_{\mathbf{P}^{n}}(b) ; \mathcal{O}_{\mathbf{P}^{n}}(d)\right)
$$

Since

$$
K_{p, q}(n, b ; d)=K_{p, q+1}(n, b-d ; d),
$$

we will always assume $0 \leqslant b \leqslant d-1$.

The following result is more precise than those in [EL12], since in that paper, $b$ was always fixed and $d \geqslant n+1$.

Theorem 2.1. Fix any $d$, any $b \in[0, d-1]$, and any $q \in[0, n+1-(n+b) / d]$. Define $m$ and $r$ as the quotient and remainder of $q d+b$ by $d-1$. Then

$$
K_{p, q}(n, b ; d) \neq 0
$$

for all $p$ in the range

$$
\left(\begin{array}{c}
m+d \\
m
\end{array}\right)-\left(\begin{array}{c}
m+d-r-1 \\
m
\end{array}\right)-m \leqslant p \leqslant\left(\begin{array}{c}
n+d \\
n
\end{array}\right)+\left(\begin{array}{c}
n-m+r \\
n-m
\end{array}\right)-\left(\begin{array}{c}
n-m+d \\
n-m
\end{array}\right)-m-1 .
$$

If $q \notin[0, n+1-(n+b) / d]$, then $K_{p, q}(n, b ; d)$ is automatically zero; see Remark 2.6. On the other hand, if $d \geqslant n+b$, then the nonvanishing holds for all $0 \leqslant q \leqslant n$.

For the proof, the idea is to work modulo a regular sequence to arrive at a situation where we can work by hand with monomials. Specifically, by the technique of Artinian reduction, we can compute syzygies modulo a linear regular sequence. Having fixed $d>0$, we put

$$
\bar{S} \stackrel{\text { def }}{=} S /\left(x_{0}^{d}, \ldots, x_{n}^{d}\right) \text {. }
$$

Slightly abusively, we view $\bar{S}$ as the graded ring spanned by monomials in the $x_{i}$ in which all variables have exponent at most $d$, with multiplication determined by the vanishing of the $d$ th 


\section{A QUICK PROOF OF NONVANISHING}

power of each variable.

Since $x_{0}^{d}, \ldots, x_{n}^{d}$ is a linear regular sequence in $\operatorname{Sym}\left(S_{d}\right)$, working modulo these powers does not affect the Koszul cohomology groups. In other words,

$$
K_{p, q}^{\operatorname{Sym}\left(S_{d}\right)}\left(S(b)^{(d)}\right) \cong K_{p, q}^{\operatorname{Sym}\left(\bar{S}_{d}\right)}\left(S(b)^{(d)} \otimes_{\operatorname{Sym}\left(S_{d}\right)} \operatorname{Sym}\left(\bar{S}_{d}\right)\right) \cong K_{p, q}^{\operatorname{Sym}\left(\bar{S}_{d}\right)}\left(\bar{S}(b)^{(d)}\right) .
$$

It thus suffices to compute this last group, which is the homology at the middle of

$$
\bigwedge^{p+1} \bar{S}_{d} \otimes \bar{S}_{(q-1) d+b} \stackrel{\partial_{p+1}}{\longrightarrow} \bigwedge^{p} \bar{S}_{d} \otimes \bar{S}_{q d+b} \stackrel{\partial_{p}}{\longrightarrow} \bigwedge^{p-1} \bar{S}_{d} \otimes \bar{S}_{(q+1) d+b} .
$$

In particular, $K_{p, q}(n, b ; d) \neq 0$ if and only if this complex has nontrivial homology, and we are therefore reduced to studying cycles and boundaries in (2.1).

We start with some notation that will prove useful. Fix a finite set of elements $P \subseteq \bar{S}$ (which in practice will be a collection of monomials).

Definition 2.2. We write $\zeta \in \bigwedge P$ (or $\left.\zeta \in \bigwedge^{s} P\right)$ if

$$
\zeta=m_{1} \wedge \cdots \wedge m_{s}
$$

with $m_{i} \in P$ for all $i$. We write $\zeta=\operatorname{det} P$ if $\zeta$ is the wedge product of all elements in $P$ (in some fixed order). We say that a wedge product $m_{1} \wedge \cdots \wedge m_{s}$ is a monomial if each $m_{i}$ is a monomial.

The following lemma guarantees the existence of many nonzero monomial classes in the cohomology of (2.1). It systematizes the computations worked out for a special case in the introduction.

Lemma 2.3. Fix a nonzero monomial $f \in \bar{S}_{q d+b}$, and denote by

$$
Z_{f}, D_{f} \subseteq \bar{S}_{d}
$$

respectively the set of degree $d$ monomials that annihilate or divide $f$.

(i) If $\zeta \in \bigwedge^{p} Z_{f}$, then $\zeta \otimes f \in \operatorname{ker} \partial_{p}$.

(ii) Let $\zeta \in \bigwedge^{s} \bar{S}_{d}$ be any monomial such that $\operatorname{det} D_{f} \wedge \zeta \otimes f$ is nonzero. Then

$$
\left(\operatorname{det} D_{f} \wedge \zeta\right) \otimes f \notin \operatorname{im} \partial_{\left(\left|D_{f}\right|+s\right)} \text {. }
$$

Proof. For statement (i), write $\zeta=m_{1} \wedge \cdots \wedge m_{s}$ with $m_{i} \in Z_{f}$. Since $m_{i} f=0 \in \bar{S}$ for all $i=1, \ldots, s$, the assertion is immediate. Turning to statement (ii), assume

$$
\partial\left(\sum \xi_{j} \otimes g_{j}\right)=\left(\operatorname{det} D_{f} \wedge \zeta\right) \otimes f
$$

Then there must be some index $j$ and some monomial appearing in $\xi_{j} \otimes g_{j}$ that maps to the monomial $\left(\operatorname{det} D_{f} \wedge \zeta\right) \otimes f$. In particular, $\xi_{j} \otimes g_{j}$ must contain a nonzero monomial of the form $\left(m \wedge \operatorname{det} D_{f} \wedge \zeta\right) \otimes g$, where $m g=f$. But then $m \in D_{f}$ and hence $m \wedge \operatorname{det} D_{f}=0$, giving a contradiction.

Corollary 2.4. Given $q$, $d$, and $b$, let $f \in \bar{S}_{q d+b}$ be a monomial such that $D_{f} \subseteq Z_{f}$. Then any nonzero monomial of the form

$$
\left(\operatorname{det} D_{f} \wedge \zeta\right) \otimes f
$$

where $\zeta \in \bigwedge Z_{f}$, represents a nonzero element of the cohomology of (2.1). In particular,

$$
K_{p, q}(n, b ; d) \neq 0
$$

for every $p$ satisfying

$$
\left|D_{f}\right| \leqslant p \leqslant\left|Z_{f}\right|
$$




\section{Ein, D. ERman and R. LAzArsfeld}

Remark 2.5. The Koszul classes just constructed are linearly independent. In fact, keeping the notation of the corollary, and with an appropriate degree twist, there is a natural map from the Koszul complex on the linear forms in $Z_{f}$ to the minimal free resolution of $\bar{S}(b)^{(d)}$ over $\operatorname{Sym}\left(\bar{S}_{d}\right)$ given by $1 \mapsto f$. This induced map yields an inclusion of the Koszul subcomplex on the linear forms

$$
Z_{f} \backslash D_{f} \subseteq \operatorname{Sym}\left(\overline{S_{d}}\right)
$$

spanning homological degrees $p=\left|D_{f}\right|,\left|D_{f}\right|+1, \ldots,\left|Z_{f}\right|$. In Conjecture B from [EEL15], we conjectured that each row of the Betti table of a high degree Veronese variety looks roughly like the Betti table of a Koszul complex. Although this result has a similar flavor, the lower bound on the size of the Koszul cohomology groups constructed via this method is far too small to verify the conjecture of [EEL15].

Theorem 2.1 now follows from Corollary 2.4 by choosing a convenient monomial $f$ and computing the number of elements in the resulting sets $Z_{f}$ and $D_{f}$.

Proof of Theorem 2.1. Put

$$
s_{d}=\operatorname{dim} \bar{S}_{d}=\left(\begin{array}{c}
n+d \\
d
\end{array}\right)-(n+1) .
$$

Let $f$ be the "leftmost" monomial of $\bar{S}$ having degree $d q+b$; by our definition of $m$ and $r$ this is the monomial of the form

$$
f=x_{0}^{d-1} \cdot x_{1}^{d-1} \cdot \ldots \cdot x_{m-1}^{d-1} \cdot x_{m}^{r} .
$$

In order to establish the theorem, it suffices to prove three assertions:

(i) $s_{d}-\left|Z_{f}\right|=\left(\begin{array}{c}d+n-m \\ d\end{array}\right)-\left(\begin{array}{c}n+r-m \\ r\end{array}\right)-n+m-2$.

(ii) $\left|D_{f}\right|=\left(\begin{array}{c}m+d \\ m\end{array}\right)-\left(\begin{array}{c}m+d-r-1 \\ m\end{array}\right)-m$.

(iii) $D_{f} \subseteq Z_{f}$.

For assertion (i), observe that $Z_{f}=(0: \bar{S} f)_{d}$ contains all monomials of degree $d$ that are divisible by any of $x_{0}, \ldots, x_{m-1}$ as well as those divisible by $x_{m}^{r}$. Hence among the $s_{d}$ monomials in $\bar{S}_{d}$, the ones not lying in $Z_{f}$ are the monomials of degree $d$ appearing in the quotient

$$
\bar{S} /\left(x_{0}, \ldots, x_{m-1}, x_{m}^{d-r}\right) .
$$

We can compute this via the resolution

$$
\ldots \longrightarrow \frac{\bar{S}(-d)}{\left(x_{0}, \ldots, x_{m-1}\right)} \stackrel{x_{m}^{r}}{\longrightarrow} \frac{\bar{S}(-d+r)}{\left(x_{0}, \ldots, x_{m-1}\right)} \stackrel{x_{m}^{d-r}}{\longrightarrow} \frac{\bar{S}}{\left(x_{0}, \ldots, x_{m-1}\right)} \longrightarrow \frac{\bar{S}}{\left(x_{0}, \ldots, x_{m-1}, x_{m}^{d-r}\right)} .
$$

Therefore

$$
\begin{aligned}
s_{d}-\left|Z_{f}\right| & =\operatorname{dim}_{k}\left(\bar{S} /\left(x_{0}, \ldots, x_{m-1}, x_{m}^{d-r}\right)\right)_{d} \\
& =\operatorname{dim}\left(\bar{S} /\left(x_{0}, \ldots, x_{m-1}\right)\right)_{d}-\operatorname{dim}\left(\bar{S} /\left(x_{0}, \ldots, x_{m-1}\right)\right)_{r}+\operatorname{dim}\left(\bar{S} /\left(x_{0}, \ldots, x_{m-1}\right)\right)_{0} \\
& =\left(\left(\begin{array}{c}
d+n-m \\
d
\end{array}\right)-n+m-1\right)-\left(\begin{array}{c}
n+r-m \\
r
\end{array}\right)+1 .
\end{aligned}
$$

For assertion (ii), observe that the set $D_{f}$ can be identified with the degree $d$ monomials of 


\section{A QUICK PROOF OF NONVANISHING}

$\bar{S} /\left(x_{m}^{r+1}, x_{m+1}, \ldots, x_{n}\right)$. A similar computation yields

$$
\begin{aligned}
\left|D_{f}\right| & =\operatorname{dim}\left(\bar{S} /\left(x_{m}^{r+1}, x_{m+1}, \ldots, x_{n}\right)\right)_{d} \\
& =\operatorname{dim}\left(\bar{S} /\left(x_{m+1}, \ldots, x_{n}\right)\right)_{d}-\operatorname{dim}\left(\bar{S} /\left(x_{m+1}, \ldots, x_{n}\right)\right)_{d-r-1}+\operatorname{dim}\left(\bar{S} /\left(x_{m+1}, \ldots, x_{n}\right)\right)_{0} \\
& =\left(\left(\begin{array}{c}
m+d \\
d
\end{array}\right)-m-1\right)-\left(\begin{array}{c}
d-r+m-1 \\
m
\end{array}\right)+1 .
\end{aligned}
$$

Finally, since the exponent of $x_{m}$ in $f$ is $r \leqslant d-1$, it follows that any element of $D_{f}$ is divisible at least by one of $x_{0}, \ldots, x_{q-1}$, and hence any such element annihilates $f$.

Remark 2.6. If $q<0$, then since $b \geqslant 0$, we clearly have $K_{p, q}(n, b ; d)=0$ for all $p$. If $q>$ $n+1-(n+b) / d$, then we claim that we also get vanishing for all $p$. We define $q^{\prime}:=n+1-q$ and note that the above inequality on $q$ is equivalent to having $q^{\prime} d<n+b$. We then use duality to compute

$$
K_{p, q}(n, b ; d)^{*}=K_{r_{d}-n-p, q^{\prime}}(n,-n-1-b ; d) .
$$

Since $\mathcal{O}\left(-n-1-b+q^{\prime} d\right)$ has no sections when $q^{\prime} d<n+1+b$, our assumptions imply that this group equals zero for all $r_{d}-n-p \geqslant 0$ and hence for all $p \geqslant 0$.

Remark 2.7. Zhou [Zho15] has recently established some results about the asymptotic distribution of torus weights appearing in the $K_{p, q}$ of toric varieties. It would be interesting to know if the present arguments can be used to give more refined information in the case $X=\mathbf{P}^{n}$.

Remark 2.8. It is conjectured in [EL12, Conjecture 7.5] that for $d \geqslant n+1$, the assertion of Theorem 2.1 is optimal in the sense that the $K_{p, q}$ in question vanish outside the stated range, and we conjecture that the more general bounds in Theorem 2.1 are optimal as well.

For instance, in the case $d=2$ and $b=0$, the full resolution is known in characteristic zero by work of Józefiak, Pragacz, and Weyman in [JPW81]. Their theorem shows that as long as $n+1 \geqslant 2 q$, we have $K_{p, q}(n, 0 ; 2)=0$ starting with $p=2 q^{2}-q$, and this value lines up with the lower bound in Theorem 2.1.

It would be exceedingly interesting to know whether one can use the approach introduced here to make progress on this conjecture, at least in the case $d \gg 0$ as in [EL12, Problem 7.7]. Unfortunately, it seems that one cannot work only with monomials - it is possible for instance that a monomial Koszul cocycle appears as the boundary of nonmonomial elements. It is tempting to wonder whether there are Gröbner-like techniques that could be used to study the issue systematically. We note that Raicu [Rai12] has reduced the general vanishing conjecture [EL12, Conjecture 7.1] to the case of Veronese syzygies.

\section{Nonvanishing for arithmetically Cohen-Macaulay schemes}

In this section we extend the results of the previous section to the setting of arithmetically Cohen-Macaulay (ACM) schemes.

Consider an arithmetically Cohen-Macaulay scheme $X \subseteq \mathbf{P}^{m}$ of dimension $n$ over the field $\mathbf{k}$, and let

$$
R=\oplus H^{0}\left(X, \mathcal{O}_{X}(k)\right)
$$

be the homogeneous coordinate ring of $X$. Setting $L_{d}=\mathcal{O}_{X}(d)$ and $B=\mathcal{O}_{X}(b)$, we are interested in the syzygies

$$
K_{p, q}\left(X, B ; L_{d}\right)=K_{p, q}\left(R(b)^{(d)}\right)
$$




\section{Ein, D. ERman And R. LAZARSfeld}

of $B$ with respect to $L_{d}$ for $d \gg 0$. Put

$$
c=c(X)=\min \left\{k \mid H^{n}\left(X, \mathcal{O}_{X}(k-n)\right)=0\right\},
$$

and write

$$
r_{d}=\operatorname{dim} H^{0}\left(X, \mathcal{O}_{X}(d)\right)=\operatorname{dim} R_{d}, \quad r_{d}^{\prime}=r_{d}-(\operatorname{deg} X)(n+1) .
$$

Our first result holds when $d \geqslant b+q+c+1$.

Theorem 3.1. Fix $b \in[0, d-q-1-c]$.

(i) If $q \in[1, n-1]$, then $K_{p, q}\left(X, B ; L_{d}\right) \neq 0$ for

$$
(\operatorname{deg} X)(q+b+1)\left(\begin{array}{c}
d+q-1 \\
q-1
\end{array}\right) \leqslant p \leqslant r_{d}^{\prime}-(\operatorname{deg} X)(d-q-b)\left(\begin{array}{c}
d+n-q-1 \\
n-q-1
\end{array}\right) .
$$

(ii) When $q=n$, one has $K_{p, n}\left(X, B ; L_{d}\right) \neq 0$ when

$$
(\operatorname{deg} X)(n+b+1)\left(\begin{array}{c}
d+n-1 \\
n-1
\end{array}\right) \leqslant p \leqslant r_{d}^{\prime}-\operatorname{deg} X .
$$

(iii) When $q=0$, one has $K_{p, 0}\left(X, B ; L_{d}\right) \neq 0$ when

$$
0 \leqslant p \leqslant r_{d}^{\prime}-(d-b)\left(\begin{array}{c}
n-1+d \\
n-1
\end{array}\right) .
$$

A somewhat more complicated but sharper statement appears in Remark 3.4 below.

Remark 3.2. If we fix $b$ and $q$, we can interpret these bounds as asymptotic statements as $d \rightarrow \infty$. Under these assumptions, we are saying that $K_{p, q}\left(X, B ; L_{d}\right) \neq 0$ for all $p$ in the range

$$
\frac{\operatorname{deg}(X)(q+b+1)}{(q-1) !} d^{q-1}+O\left(d^{q-2}\right) \leqslant p \leqslant r_{d}^{\prime}-\left(\frac{\operatorname{deg}(X)}{(n-q-1) !} d^{n-q}+O\left(d^{n-q-1}\right)\right) .
$$

Conjecture 7.1 in [EL12] states that one should have $K_{p, q}=0$ for $p \leqslant O\left(d^{q-1}\right)$; it would be interesting to understand the optimal leading coefficients as well. In the ACM case this implies that $K_{p, q}=0$ also for $p>r_{d}-O\left(d^{n-q}\right)$, but in the non-ACM case the groups in question can be nonvanishing for $p \approx r_{d}$ [EL12, Remark 5.3].

For the proof of the theorem, let $I_{X} \subseteq \mathbf{k}\left[x_{0}, \ldots, x_{m}\right]$ be the defining ideal of $X$, so that $R=\mathbf{k}\left[x_{0}, \ldots, x_{m}\right] / I_{X}$. The statement is independent of the ground field, so we may assume that $\mathbf{k}$ is infinite. Then, after a general change of coordinates, we may assume that $x_{0}, \ldots, x_{n}$ form a system of parameters for $R$. To help clarify the following arguments, we will relabel the variables $x_{n+1}, \ldots, x_{m}$ as $y_{n+1}, \ldots, y_{m}$.

Let $S=\mathbf{k}\left[x_{0}, \ldots, x_{n}\right] \subseteq R$, which is a Noether normalization since $x_{0}, \ldots, x_{n}$ is a system of parameters. As $R$ is Cohen-Macaulay of dimension $n+1$, it follows that it is a maximal CohenMacaulay $S$-module, and hence a free $S$-module. We may choose a set $\Lambda$ of monomials of the form $y^{\beta} \in R$ which form a basis for $R$ as an $S$-module, so that

$$
R=\bigoplus_{y^{\beta} \in \Lambda} S \cdot y^{\beta} .
$$

We assume $1 \in \Lambda$. Thus $\operatorname{deg}(X)=\# \Lambda$, and we observe that $c(X)=\max \left\{\operatorname{deg} y^{\beta}\right\}$.

Fix $q \in[0, n], d>0$, and $b \geqslant 0$. Set

$$
\bar{R}=R /\left(x_{0}^{d}, \ldots, x_{n}^{d}\right),
$$




\section{A QUICK PROOF OF NONVANISHING}

and define $\bar{S}$ as in the previous section. Thus $\bar{R}=R \otimes_{S} \bar{S}$, and $\bar{R}$ is a free $\bar{S}$-module with basis $\Lambda$. Since $R$ is Cohen-Macaulay, we have

$$
\operatorname{dim} K_{p, q}\left(R(b)^{(d)}\right)=\operatorname{dim} K_{p, q}\left(\bar{R}(b)^{(d)}\right)
$$

for all $p$ and $q$, where the group on the right is computed as the cohomology of the complex

$$
\bigwedge^{p+1} \bar{R}_{d} \otimes \bar{R}_{(q-1) d+b} \stackrel{\partial}{\longrightarrow} \bigwedge^{p} \bar{R}_{d} \otimes \bar{R}_{q d+b} \stackrel{\partial}{\longrightarrow} \bigwedge^{p-1} \bar{R}_{d} \otimes \bar{R}_{(q+1) d+b} .
$$

In the natural way, we can speak of monomials in $\bar{R}$ : these are (the images in $\bar{R}$ of) elements of the form $x^{\alpha} y^{\beta}$, where $y^{\beta} \in \Lambda$, and the degree of such a monomial is $|\alpha|+|\beta|$. Given a monomial $f \in \bar{S}$, we denote by

$$
Z_{f}, E_{f} \subseteq \bar{R}_{d}
$$

respectively the set of degree $d$ monomials that annihilate $f$ and the collection of degree $d$ monomials of the form $x^{\alpha} y^{\beta}$, where $x^{\alpha}$ divides $f$ and $y^{\beta} \in \Lambda$.

We start with an analogue of Lemma 2.3.

LEMMA 3.3. Let

$$
f \in \bar{S}_{q d+b} \subseteq \bar{R}_{q d+b}
$$

be a monomial such that $E_{f} \subseteq Z_{f}$. Then any nonzero monomial element

$$
m=\left(\operatorname{det} E_{f} \wedge \zeta\right) \otimes f
$$

with $\zeta \in \bigwedge Z_{f}$ represents a nonzero Koszul cohomology class. In particular

$$
K_{p, q}\left(X, \mathcal{O}_{X}(b) ; \mathcal{O}_{X}(d)\right) \neq 0
$$

for every $p$ with

$$
\left|E_{f}\right| \leqslant p \leqslant\left|Z_{f}\right|
$$

Proof. Since $E_{f} \subseteq Z_{f}$, the element $m$ is evidently a Koszul cycle. It remains to prove that it is not cohomologous to zero. In fact, we will show that $m$ cannot occur as a monomial appearing in the expansion (with respect to the chosen basis of $\bar{R}$ ) of $\partial(\xi \otimes g)$ for any monomials $\xi \in \Lambda^{p+1} \bar{R}_{d}$ and $g \in \bar{R}_{(q-1) d+b}$. Suppose to the contrary that $m$ appears as a term in $\partial\left(\xi_{0} \wedge \cdots \wedge \xi_{p} \otimes g\right)$. Then after possibly reindexing and introducing a sign, we can suppose

$$
\xi_{1} \wedge \cdots \wedge \xi_{p}=\operatorname{det}\left(E_{f}\right) \wedge \zeta
$$

and that $f$ appears as a term in the expansion of $\xi_{0} g$ in terms of the basis $\Lambda$. Suppose

$$
\xi_{0}=x^{\alpha} y^{\beta}, \quad g=x^{\gamma} y^{\delta},
$$

where $y^{\beta}, y^{\delta} \in \Lambda$. Then in $\bar{R}$ we can rewrite

$$
y^{\beta+\delta}=h_{0} \cdot 1+\sum_{y^{\lambda} \in \Lambda} h_{\lambda} \cdot y^{\lambda},
$$

where $h_{\lambda} \in \bar{S}_{|\beta|+|\delta|-|\lambda|}$. Therefore $f=x^{\alpha+\gamma} h_{0}$, and consequently $x^{\alpha} y^{\beta} \in E_{f}$. In particular $\xi_{0}$ also appears as one of $\xi_{1}, \ldots, \xi_{p}$, and hence $m=0$.

We now turn to the proof of Theorem 3.1.

Proof of Theorem 3.1. As before, we take $f$ to be the "leftmost" nonzero monomial of $\bar{S}$ of degree $d q+b:$

$$
f=x_{0}^{d-1} \cdot x_{1}^{d-1} \cdot \ldots \cdot x_{q-1}^{d-1} \cdot x_{q}^{q+b}
$$




\section{Ein, D. ERman and R. LAzArsfeld}

We claim first of all that $E_{f} \subseteq Z_{f}$ provided that $d \geqslant b+q+c+1$. In fact, suppose

$$
w=x_{0}^{a_{0}} \cdot \ldots \cdot x_{q}^{a_{q}} \cdot y^{\beta} \in E_{f} .
$$

Then $a_{q} \leqslant q+b$, and hence

$$
a_{0}+\cdots+a_{q-1}=d-a_{q}-|\beta| \geqslant d-(q+b)-c>0 .
$$

Therefore at least one of $a_{0}, \ldots, a_{q-1}$ is strictly positive, and consequently $w \in Z_{f}$.

In order to apply Lemma 3.3, it remains to estimate the sizes of $E_{f}$ and $Z_{f}$. Writing $\bar{r}_{d}=$ $\operatorname{dim} \bar{R}_{d}$, we start by giving an upper bound on $\bar{r}_{d}-\left|Z_{f}\right|$. Assume first $q \in[1, n-1]$, and consider a monomial $x^{\alpha}=x_{0}^{a_{0}} \cdot \ldots \cdot x_{n}^{a_{n}}$. Then a degree $d$ monomial $x^{\alpha} y^{\beta}$ lies in the complement of $Z_{f}$ if and only if

$$
a_{0}=\cdots=a_{q-1}=0, \quad a_{q} \leqslant d-b-q-1 .
$$

The number of possibilities for $x^{\alpha}$ is (rather wastefully) bounded above simply by the number of degree $d$ monomials in $\mathbf{k}\left[x_{q+1}, \ldots, x_{n}\right]$, times the number of choices for $a_{q}$, times the number of choices for $y^{\beta}$. Since $|\Lambda|=\operatorname{deg} X$, this leads to the lower bound

$$
\bar{r}_{d}-(\operatorname{deg} X)(d-q-b)\left(\begin{array}{c}
d+n-q-1 \\
n-q-1
\end{array}\right) \leqslant\left|Z_{f}\right| .
$$

Turning to an upper bound on $\left|E_{f}\right|$, observe that $x^{\alpha} y^{\beta} \in E_{f}$ if and only if

$$
a_{0}, \ldots, a_{q-1} \leqslant d-1, \quad a_{q} \leqslant q+b \quad \text { and } \quad a_{q+1}=\cdots=a_{n}=0 .
$$

We can bound this (again wastefully) by the number of monomials of degree $d$ in $\mathbf{k}\left[x_{0}, \ldots, x_{q-1}\right]$, times the number of choices for $a_{q}$, times the number of choices for $y^{\beta}$. This leads to:

$$
(\operatorname{deg} X)(q+b+1)\left(\begin{array}{c}
q-1+d \\
q-1
\end{array}\right) \geqslant\left|E_{f}\right| .
$$

So to obtain assertion (i) of Theorem 3.1, it remains only to observe that

$$
\begin{aligned}
\bar{r}_{d} & =\sum_{y^{\beta} \in \Lambda} \operatorname{dim} \bar{S}_{d-|\beta|} \geqslant \sum_{y^{\beta} \in \Lambda}\left(\operatorname{dim} S_{d-|\beta|}-(n+1)\right) \\
& =\operatorname{dim} R_{d}-|\Lambda|(n+1)=r_{d}^{\prime} .
\end{aligned}
$$

When $q=n$ we get the same bound on $\left|E_{f}\right|$, but now we find that

$$
\bar{r}_{d}-(\operatorname{deg} X) \leqslant\left|Z_{f}\right|,
$$

and this yields statement (ii) of the theorem. Finally, when $q=0$ we get the same lower bound on $\left|Z_{f}\right|$ as above, and we can obtain nonvanishing starting with $p=0$.

Remark 3.4. By separating the estimates into two terms depending on whether $\beta$ is equal to zero or not, one gets a slightly better upper bound on the size of $E_{f}$ when $q \in[1, n-1]$ :

$$
(\operatorname{deg} X-1)(q+b+1)\left(\begin{array}{c}
q-1+d-1 \\
q-1
\end{array}\right)+\left(\begin{array}{c}
q+d \\
q
\end{array}\right)-\left(\begin{array}{c}
d-b-1 \\
q
\end{array}\right)-q \geqslant\left|E_{f}\right| .
$$

In particular, this reduces to the statements obtained for $\mathbf{P}^{n}$ in the previous sections.

Remark 3.5. By defining $m$ and $r$ as respectively the quotient and remainder of $d q+b$ by $d-1$, one can use an argument along the lines of the proof of Theorem 2.1 to extend this to some additional values of $q, d$, and $b$. However, we felt the asymptotic behavior was more clear when phrased in terms of $q$ and $b$ instead of $r$ and $m$. 


\section{A QUICK PROOF OF NONVANISHING}

Remark 3.6. The bounds for $\left|E_{f}\right|$ and $\bar{r}_{d}-\left|Z_{f}\right|$ appearing in the proof of Theorem 3.1 could be improved by a more precise count of the relevant possibilities, in particular taking into account the degrees of the $y^{\beta}$. This amounts to computations involving the numerator of the Hilbert series of $R$ (that is, the Hilbert function of the Artinian reduction $\bar{R}$ ), and confronted with a specific example, it is often quite easy to use directly the method of the proof to get stronger statements. For example, let $X \subseteq \mathbf{P}^{5}$ be the hypersurface

$$
x_{0}^{3}+\cdots+x_{5}^{3}=0 .
$$

Then $\Lambda=\left\{1, x_{5}, x_{5}^{2}\right\}$, so $c=2$. We take $(q, b, d)=(3,0,8)$ and

$$
f=x_{0}^{7} x_{1}^{7} x_{2}^{7} x_{3}^{3} \text {. }
$$

Then $R=\mathbf{k}\left[x_{0}, \ldots, x_{5}\right] /\left(x_{0}^{3}+\cdots+x_{5}^{3}\right)$ and $\bar{R}=R /\left(x_{0}^{8}, \ldots, x_{4}^{8}\right)$. The bounds from Theorem 3.1 and Remark 3.5 yield the nonvanishing result $K_{p, 3}\left(X ; \mathcal{O}_{X}(8)\right) \neq 0$ for $p$ between 540 and 1005 .

However, if we follow the method of the proof, we can compute the size of $E_{f}$ directly. Let $A:=\mathbf{k}\left[x_{0}, \ldots, x_{q}\right] /\left(x_{0}^{d}, \ldots, x_{q-1}^{d}, x_{q}^{q+b+1}\right)=\mathbf{k}\left[x_{0}, \ldots, x_{3}\right] /\left(x_{0}^{8}, x_{1}^{8}, x_{2}^{8}, x_{3}^{4}\right)$. Then

$$
\sum_{y^{\beta} \in \Lambda} \operatorname{dim} A_{d-\operatorname{deg} y^{\beta}}=\operatorname{dim} A_{8}+\operatorname{dim} A_{7}+\operatorname{dim} A_{6}=301 .
$$

A similar computation shows that there are 14 monomials in the complement of $Z_{f}$ and so $\left|Z_{f}\right|=1030-14=1016$, and the nonvanishing statement can be extended to all values of $p$ between 301 and 1016 .

Remark 3.7. Let $X \subseteq \mathbf{P}^{m}$ be an arbitrary variety of dimension $n$, and suppose that $B$ is a line or vector bundle on $X$ with the property that

$$
H^{i}\left(X, B \otimes \mathcal{O}_{X}(k)\right)=0
$$

for all $k \in \mathbf{Z}$ and $0<i<n$; in other words, $M=\oplus H^{0}\left(X, B \otimes \mathcal{O}_{X}(k)\right)$ is a Cohen-Macaulay module over the homogeneous coordinate ring of $\mathbf{P}^{m}$. Replacing $B$ by a twist, one can assume without loss of generality that $M_{-1}=0$ but $M_{0} \neq 0$. Then one can use the methods of this section to obtain effective nonvanishing statements for the syzygies $K_{p, q}\left(X, B ; \mathcal{O}_{X}(d)\right)$. In fact, the hypotheses on $M$ imply that it has a generator in degree zero, and then in the arguments above one can replace $R$ by $M$. We leave the details to the interested reader. It would be interesting to compare the resulting statements with the results [Zho14] of Zhou which fall under this rubric.

Finally, we expect that nonvanishing statements similar to Theorem 3.1 hold for any finitely generated, graded k-algebra $R$. More precisely, we conjecture the following analogue of part (i) of Theorem 3.1.

Conjecture 3.8. Fix $b$ and $R$ and $q \in[1, n]$, where $n:=\operatorname{dim} R-1$. Then there exist constants $c$ and $C$ such that if $d \gg 0$, then

$$
K_{p, q}\left(R(b)^{(d)}\right) \neq 0 \quad \text { for all } c d^{q-1} \leqslant p \leqslant r_{d}-C d^{n-q} .
$$

We expect similar analogues of parts (ii) and (iii) of Theorem 3.1, as well as analogues of the cases where $b$ is close to $d$, as in Remark 3.5.

\section{ACKNOWLEDGEMENTS}

We wish to thank Xin Zhou for valuable discussions, and the referee for some suggestions which 


\section{Ein, D. ERman and R. LAzArsfeld}

significantly streamlined the statement of Theorem 2.1.

\section{REFERENCES}

BCR11a W. Bruns, A. Conca, and T. Römer, Koszul cycles, in Combinatorial Aspects of Commutative Algebra and Algebraic Geometry, Abel Symp., vol. 6, (Springer, Berlin, 2011), 17-33; http: //dx.doi.org/10.1007/978-3-642-19492-4_2.

BCR11b _ Koszul homology and syzygies of Veronese subalgebras, Math. Ann. 351 (2011), no. 4, 761-779; http://dx.doi.org/10.1007/s00208-010-0616-1.

CJKW14 A. Conca, M. Juhnke-Kubitzke, and W. Welker, Asymptotic syzygies of Stanley-Reisner rings of iterated subdivisions, arXiv:1411.3695.

EEL15 L. Ein, D. Erman, and R. Lazarsfeld, Asymptotics of random Betti tables, J. reine angew. Math. 702 (2015), 55-75; http://dx.doi.org/10.1515/crelle-2013-0032.

EGHP05 D. Eisenbud, M. Green, K. Hulek, and S. Popescu, Restricting linear syzygies: algebra and geometry, Compos. Math. 141 (2005), no. 6, 1460-1478; http://dx.doi.org/10.1112/ S0010437X05001776.

EL12 L. Ein and R. Lazarsfeld, Asymptotic syzygies of algebraic varieties, Invent. Math. 190 (2012), no. 3, 603-646; http://dx.doi.org/10.1007/s00222-012-0384-5.

JPW81 T. Józefiak, P. Pragacz, and J. Weyman, Resolutions of determinantal varieties and tensor complexes associated with symmetric and antisymmetric matrices, Young Tableaux and Schur Functors in Algebra and Geometry (Torun, 1980), Astérisque, vol. 87 (Soc. Math. France, Paris, 1981), 109-189.

OP01 G. Ottaviani and R. Paoletti, Syzygies of Veronese embeddings, Compos. Math. 125 (2001), no. 1, 31-37; http://dx.doi.org/10.1023/A:1002662809474.

Rai12 C. Raicu, Representation stability for syzygies of line bundles on Segre-Veronese varieties, arXiv:1209.1183.

Rub04 E. Rubei, A result on resolutions of Veronese embeddings, Ann. Univ. Ferrara Sez. VII (N.S.) 50 (2004), 151-165; http://dx.doi.org/10.1007/BF02825348.

Zho14 X. Zhou, Effective non-vanishing of asymptotic adjoint syzygies, Proc. Amer. Math. Soc. 142 (2014), no. 7, 2255-2264; http://dx.doi.org/10.1090/S0002-9939-2014-11947-2.

Zho15 Asymptotic weights of syzygies of toric varieties, arXiv:1501.06112.

Lawrence Ein ein@uic.edu

Department of Mathematics, University Illinois at Chicago, 851 South Morgan St., Chicago, IL 60607, USA

Daniel Erman derman@math.wisc.edu

Department of Mathematics, University of Wisconsin, Madison, WI 53706, USA

Robert Lazarsfeld robert.lazarsfeld@stonybrook.edu

Department of Mathematics, Stony Brook University, Stony Brook, NY 11794, USA 